\section{Studie zeigt Überlebensvorteil bei Brustkrebs}

Die im Februar in The Lancet [1] vorgestellten Forschungsergebnisse der Intergroup Exemestane Study (IES) zeigen: Eine adjuvante Sequenztherapie mit dem Wirkstoff Exemestan von Pfizer verbessert signifikant die Überlebenschance von postmenopausalen Frauen mit nachweislich hormonabhängigem Brustkrebs um 17\% $(p=0,04)$ im Vergleich zum bisherigen Therapiestandard [2]. An der Langzeitstudie, die im Februar 1998 begann, haben insgesamt 4742 Brustkrebspatientinnen teilgenommen.

Wenn Frauen in der Postmenopause an hormonabhängigem Brustkrebs erkranken, erhalten sie im Anschluss an eine kurative Therapie gewöhnlich fünf Jahre lang eine Antihor- monbehandlung, z.B. mit Tamoxifen, um das Risiko eines Rückfalls zu verringern und die Überlebenschancen zu verbessern. Wie die IES-Langzeitstudie nun zeigt, haben Frauen mit nachweislich hormonabhängigem Brustkrebs durch eine kombinierte Behandlung aus einer zwei- bis dreijährigen Therapie mit Tamoxifen und anschließender Umstellung auf Exemestan ein geringeres Risiko, einen Rückfall zu erleiden, als mit einer fünfjährigen alleinigen Behandlung mit dem Antiöstrogen Tamoxifen. In dem Behandlungsarm mit Exemestan wurde zudem eine Reduktion des Risikos von Fernmetastasen um 18\% (95\% CI, 0.69-0.98) sowie eine Verringerung des Risikos eines kontralateralen Mammakarzinoms um 44\% (95\% CI, 0.33-0.98) beobachtet [1]. Die IES ist die erste und einzige doppelblinde, randomisierte und multizentrische Studie zur adjuvanten Sequenztherapie und verfügt über einen medianen Nachbeobachtungszeitraum von 56 Monaten.

\section{Literatur}

1 Coombes et al, Randomised Trial of Exemestane versus Tamoxifen after 2-3 years Tamoxifen Therapy: Survival and safety in the Intergroup Exemestane Study, Lancet 2007; Published online Feb 13, 2007. DOI: 10.1016/S0140-6736(07)60200-1.

2 Bliss JM, ASCO 2006, oral presentation, Abstract \# LBA 527.

Weitere Informationen bei

Haas \& Health Partner

Public Relations GmbH

Lars Bruhn

Große Hub 10 c

65344 Eltville

Tel. +49 6123 7057-51, Fax -57

bruhn@haas-health.de
Studienteilnehmerinnen in Deutschland gesucht

\section{Gebärmutterhalskrebs: \\ Phase-II-Studien mit multi-target Tyrosinkinase-Inhibitoren gestartet}

Zur potenziellen Behandlung von Gebärmutterhalskrebs (Zervixkarzinom) hat GlaxoSmithKline (GSK) klinische Phase-II-Studien gestartet. Die internationalen, multizentrischen Studien untersuchen die Wirksamkeit und Verträglichkeit der Wirkstoffkombination Lapatinib + Pazopanib bei der Behandlung von fortgeschrittenem Gebärmutterhalskrebs. Vorangegangene Studien haben gezeigt, dass Pazopanib das Wachstum solider Tumore hemmen kann, indem der Wirkstoff die Blutversorgung des Tumors drosselt. An der Studie sollen über einen Zeitraum von 12
Monaten insgesamt 180 Frauen mit fortgeschrittenem Gebärmutterhalskrebs teilnehmen. 60 Frauen werden ausschließlich Pazopanib, 60 Lapatinib und weitere 60 eine Kombination aus beiden Wirkstoffen in Tablettenform erhalten.

\section{Patientinnen in Deutschland gesucht}

Für die Studie werden in Deutschland noch Studienteilnehmerinnen gesucht, die unter anderem mindestens 18 Jahre alt sind und sich von den Auswirkungen einer früheren Operation oder Chemotherapie erholt haben. Eine vorangegangene Chemotherapie muss bei Studienbeginn mindestens drei Wochen zurückliegen. Außerdem müssen mögliche Interessentinnen Tabletten einnehmen können. Die Teilnehmerinnen der Studie werden nach einer ausführlichen Voruntersuchung per Zufallsentscheid entweder einmal täglich Pazopanib oder Lapatinib oder eine Kombination aus den beiden Medikamenten einnehmen.

Unter www.tyrosinkinasehemmer.de sind weitere Informationen $\mathrm{zu}$ finden. Patientinnen und Ärzte, die an der Studie teilnehmen möchten, können sich direkt bei GlaxoSmithKline unter der Servicenummer 01803456100 (Patientinnen) und 0800-1223355 (Ärzte) melden.

Weitere Informationen bei

GlaxoSmithKline GmbH \& Co. KG

Arnd Prilipp

Theresienhöhe 11

80339 München

Tel. +49 89 36044-8117, Fax -98117

Arnd.Prilipp@gsk.com

\title{
PharmaTicker+++ PharmaTicker+++ PharmaTicker+++ PharmaTicker+++
}

Baxter Deutschland GmbH. Soeben wurde das neue Programmheft des Baxter Competence Centers veröffentlicht. Auch im Jahr 2007 steht eine große Vielfalt an Fortbildungen aus unterschiedlichsten Fachgebieten für Ärzte, Apotheker und Pflegekräfte zur Verfügung. Das komplette Programmheft kann bestellt werden unter baxter@the-messengers.de.

Anmeldung zu den Veranstaltungen unter

Tel. +496172 2851014

baxter@the-messengers.de

www.baxter.de
B. Braun Melsungen AG. Mit Softa-Man acute bietet die B. Braun Melsungen AG jetzt aktuell ein Händedesinfektionsmittel mit einer umfassenden viruziden Wirksamkeit von einer Minute an. Es ist damit sehr schnell wirksam, sicher und anwenderfreundlich. Der Wirkstoffgehalt von Softa-Man acute ist mit $63 \%$ wesentlich niedriger als von herkömmlichen Präparaten. Außerdem ist es parfüm- und farbstofffrei.

Weitere Informationen bei

B. Braun Melsungen AG

Hotline 0800-2272868

kundenservice@bbraun.com
Sanofi Pasteur MSD GmbH. Die Ständige Impfkommission (STIKO) am Robert Koch-Institut in Berlin hat die Impfung gegen humane Papillomviren (HPV) als Standardimpfung empfohlen und damit in den Impfkatalog aufgenommen. Gardasil $^{\circledR}$ von Sanofi Pasteur MSD ist bislang der einzige in Deutschland zugelassene Impfstoff zur Prävention von Gebärmutterhalskrebs und anderen HPV-assoziierten Erkrankungen.

Weitere Informationen bei

Sanofi Pasteur MSD GmbH

Michael Kölsch

Tel. +49 6224 594-233, Fax -161 MKoelsch@spmsd.com 